\title{
Las patentes como indicador de la actividad científica en las universidades españolas
}

\author{
Por Francisco-Javier Martínez-Méndez, Juan-Antonio Pastor-Sánchez y Rosana López-Carreño
}

\begin{abstract}
Resumen: Los estudios de la actividad científica española discriminan habitualmente las patentes como indicador relevante, centrándose más en las publicaciones en revistas recogidas en los índices de impacto. Esta realidad contrasta con las tesis de la literatura especializada, que muestra a las patentes como uno de los indicadores de mayor importancia para medir la productividad científica. Se ha realizado un caso de estudio sobre las patentes registradas por las universidades españolas durante 2008, tomando como fuente la base de datos European Patents Fulltext, analizando su distribución por instituciones universitarias en este período de tiempo. Se observa una cierta correlación con otros

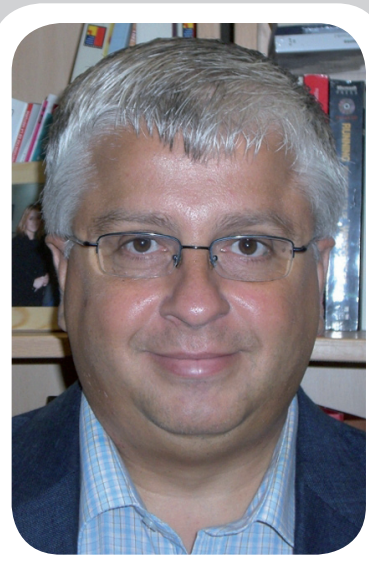

Francisco-Javier MartínezMéndez es doctor en documentación por la Universidad de Murcia y profesor de tecnologías de la información en la Facultad de Comunicación y Documentación. Principalmente lleva a cabo su trabajo en el campo de la recuperación de información en la Web.

http://irsweb.es
\end{abstract} indicadores, dato que puede

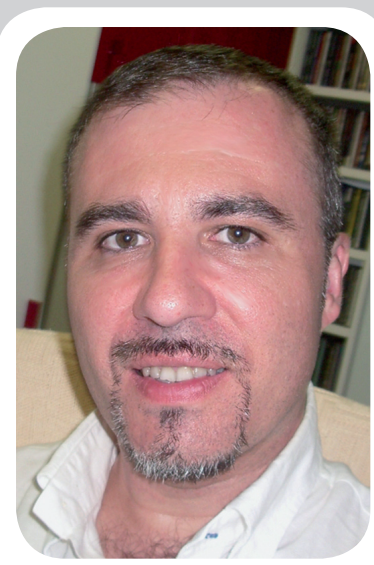

Juan-Antonio Pastor-Sánchez es doctor en documentación por la Univ. de Murcia y profesor de construcción de sistemas de información digital en la Fac. de Comunicación y Documentación. Trabaja en organización del conocimiento y representación de información en la Web, en especial tesauros colaborativos por medio de SKOS.

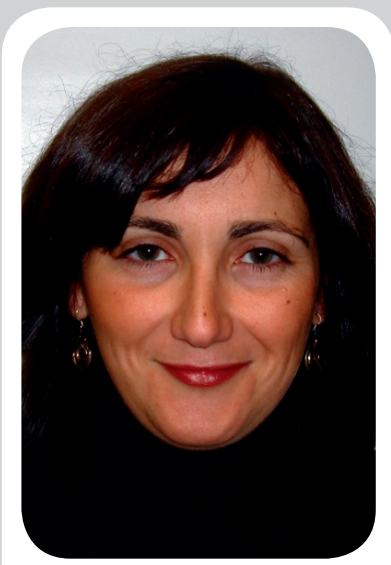

Rosana López-Carreño es doctora en documentación por la Universidad de Murcia y profesora de fuentes de información especializadas en la Facultad de Comunicación y Documentación. Principalmente desarrolla su trabajo en este campo y en el de la documentación educativa.

servir de base para plantear que las patentes pueden y deben ser consideradas en la realización de este tipo de análisis.

Palabras clave: Patentes, Actividad científica, Estudios e informes, Universidades, España.

Title: Patents as an indicator of the scientific activity in Spanish universities

Abstract: The studies and analysis of Spanish scientific activity usually are more focused on contributions in journals with impact factor than on patents as a relevant indicator of the scientific work. This reality contrasts with the trends of the specialized literature, which show patents to be one of the most important parameters to measure scientific productivity. Therefore, we conducted a case study on the patents registered by Spanish Universities in 2008, using as our source the European Patents Fulltext database. Analysis of the distribution of patents by university in this specific period of time revealed a certain correlation with other commonly used indicators. This finding can be a basis for suggesting that patents can and should be considered when conducting such studies.

Keywords: Patents, Scientific activity, Analysis and reports, Universities, Spain.

Martínez-Méndez, Francisco-Javier; Pastor-Sánchez, Juan-Antonio; López-Carreño, Rosana. "Las patentes como indicador de la actividad científica en las universidades españolas". El profesional de la información, 2010, marzo-abril, v. 19, n. 2, pp. 168-174.

DOI: 10.3145/epi.2010.mar.07

\section{Actividad y productividad científica de las universidades españolas}

La realización de análisis sobre la actividad científica de las universidades españolas (y más concretamente de su impacto) es una línea de trabajo consolidada en los estudios métricos y ocupa, cada vez con mayor frecuencia, un hueco en los medios de comunicación social. En España se ha pasado de disponer sólo del Ranking de las 50 mejores titulaciones (elaborado anualmente por el diario El mundo a partir de una encuesta entre profesores universitarios) a consultar habitualmente trabajos muchos más elaborados como los 
de Buela-Casal et al. (2009), SCImago (2007), o Jiménez-Contreras, De-Moya-Anegón y Delgado-LópezCózar (2003). En una perspectiva mucho más global, podemos felicitarnos por disponer de una herramienta tan importante como el SCImago Journal Rank ${ }^{1}$ (SCImago, 2007) basado en la base de datos Scopus. Estas publicaciones trascienden más allá de los campus y generan debate social, algo lógico porque la sociedad sustenta a la universidad y ésta debe rendirle cuentas (más si cabe en el contexto socioeconómico actual de grave crisis económica). Es ahora más necesario fomentar la relación con empresas, mejorando la productividad y la transferencia de tecnología. Así, "las universidades se animan paulatinamente a reconocer que esta relación es de importancia estratégica y forma parte de su compromiso de servir al interés público" (European Commission, 2006). El hecho de que los grupos de investigación universitarios interactúen con empresas y otras instituciones en el registro, elaboración y comercialización de una patente puede convertirse en una oportunidad de generación de nuevos recursos financieros para las universidades (Azagra-Caro, 2009). Esta colaboración es una faceta más de la actividad científica y por tanto debería ser evaluada junto con otros indicadores, no de forma separada.

\section{"El análisis de la productividad científica en las universidades españolas comienza a aparecer en los medios"}

Estos trabajos han repercutido en la vida cotidiana de las comunidades universitarias, generando debate. Mostramos dos ejemplos: el primero tiene lugar en la Universidad de Murcia al conocerse los datos del trabajo de Buela-Casal et al. (2009), e intentar los responsables de esta institución justificar (o al menos intentarlo) el menor número de proyectos de $\mathrm{I}+\mathrm{D}+\mathrm{i}$ en curso en comparación con la vecina Universidad Politécnica de Cartagena (más pequeña y joven), alegando que el importe total de fondos concedidos era mayor y recordando el tercer puesto obtenido por sus catedráticos en el ranking de sexenios de investigación en el ámbito nacional. El segundo ejemplo acaece a finales del curso 2008-2009, cuando el rector de la Universidad Carlos III de Madrid denunció falta de rigor en un estudio realizado por Buesa para el Instituto de Análisis Industrial y Financiero de la Universidad Complutense de Madrid con datos del curso 2005-2006 y del que se hacía eco el diario El país de 25 de mayo de 2009. Esta queja por parte de la $U C 3 M$ fue inmediatamente contestada por el autor en otros medios de información abandonando el debate científico y entrando claramente en uno político.
Independientemente de otras consideraciones, estamos convencidos de la necesidad de este tipo de análisis, y estamos seguros de que contribuirán a dinamizar más el trabajo de las instituciones universitarias, algo positivo para todos.

Cada uno de estos trabajos emplea distintos indicadores y aplica enfoques particulares en la interpretación de los resultados. La publicación de artículos científicos en revistas recogidas en el ISI Web of science o, más recientemente, en la base de datos Scopus es la piedra angular de los mismos, si bien algunos trabajos informan sobre los recursos financieros dedicados a la investigación, el número de profesores e investigadores contratados, los proyectos de I+D+i, becas FPU (Formación del profesorado universitario), número de tesis doctorales y cuántos programas de doctorado poseen mención de calidad. Resulta extraño comprobar que casi todos estos trabajos (excepto el criticado informe de Buesa) obvian el uso de las patentes como indicador a pesar de ser un documento que también comunica el avance científico y resulta de gran utilidad al "especificar quién es el dueño de la nueva información creada, cuando ésta se plasma en tecnología susceptible de ser aplicada en procesos productivos" (Sáiz-González, 1999). Si bien su propósito último puede no ser la divulgación científica, no es menos cierto que contribuyen a ello (Villanueva-Kurczyn, 2008) y que junto a los tradicionales indicadores bibliométricos se aceptan "otros indicadores como patentes o nuevos productos en las áreas tecnológicas" (Bordons; Zulueta-García, 1999).

Para Spinak (1998) "la bibliometría estudia la organización de los sectores científicos y tecnológicos a partir de fuentes bibliográficas y patentes para identificar a los actores, a sus relaciones y a sus tendencias". Si las patentes no encuentran su hueco entre los autores bibliométricos, tampoco los tradicionales indicadores de impacto suelen ser tomados en consideración por los analistas de otras áreas más afines a la economía.

En el exhaustivo trabajo de González-Albo-Manglano y Zulueta-García (2007) se analizan las patentes solicitadas por las universidades españolas en el período 1980-2000 y el gasto de cada institución en I+D, no prestando atención a la producción de otros documentos científicos. Esos autores constatan una separación clara y una relegación de los análisis de patentes: "el estudio del avance científico ha centrado su atención en la evaluación de los resultados de carácter básico que se reflejan en artículos científicos y documentos similares -ponencias a congresos, reseñas, etc.--, dejando en un segundo plano la investigación aplicada o tecnológica" (González-Albo-Manglano; Zulueta-García, 2007).

La revisión bibliográfica realizada lleva a pensar en una especie de status quo: la investigación básica se 
mide por medio de índices de impacto y la investigación aplicada en términos de patentes e inversión en $\mathrm{I}+\mathrm{D}$, aunque algunos grupos aproximan ambas tendencias. Mantener en el tiempo esta separación no parece muy adecuado, máxime cuando en el trabajo de Van Looy et al. (2006) queda demostrado que los científicos "inventores" tienden a publicar mucho más que los "no inventores" entre otras cosas porque incluso varios años después de la presentación del invento se sigue observando actividad científica alrededor de la patente. Las patentes potencian la producción de publicaciones, es decir, "las actividades de investigación aplicada se relacionan con las actividades investigadoras básicas; queda ahora determinar ese grado de relación que puede llegar a producir beneficios mutuos sobre la actividad científica en aquellas áreas donde los temas de interés estén fuertemente vinculados" (Carayol, 2003).

\section{"Se ha generado un lógico debate porque la sociedad sustenta a la universidad y de alguna manera ésta debe rendirle cuentas"}

\section{Las patentes como medida de la productividad científica}

El calificar como extraordinaria la ausencia de las patentes en los análisis antes citados no es una exageración. Este tipo documental es curiosamente uno de los mecanismos más aceptados para medir la productividad científica (entendía como actividad seguida de rendimiento a partir de la transferencia de tecnología). Las patentes "aportan información relevante sobre el proceso de innovación tecnológica, al contrario que los indicadores relacionados con los gastos de $\mathrm{I}+\mathrm{D}$ que tan sólo aportan información sobre las actividades y no ofrecen una visión global de las capacidades tecnológicas disponibles por la organización" (Hidalgo-Nuchera, 2003, p. 8). La mayoría de los trabajos consultados cita a Schmookler (1966) como el precursor de su uso como indicador tecnológico y la mayoría coincide en que "es casi imposible encontrar estudios sobre los procesos de innovación o las capacidades tecnológicas de cualquier organización (empresa, universidad, centro de investigación) sin que se utilicen las patentes como indicadores asociados" (Hidalgo-Nuchera, 2003, p. 9).

Las patentes sirven también para medir el grado de colaboración entre la universidad y la empresa, aspecto que siempre se ha considerado básico para la evolución de una sociedad: "mientras que los países líderes en tecnología han alcanzado cierto grado de fluidez en la interacción universidad-empresa y en la generación de patentes universitarias y se preguntan dónde están los límites, los países rezagados tecnológicamente están más interesados en cómo promover estas tendencias. Esto se observa tanto en países donde el proceso de seguimiento está bastante avanzado, como Singapur, como en países donde no lo está, como España" (Azagra-Caro, 2004, p. 131). También es cierto que no todas las innovaciones se patentan, ya que hay métodos alternativos para proteger las invenciones o los gastos económicos asociados no justifican la necesidad, sin llegar a olvidar que en muchos casos no se persigue beneficio económico alguno en la investigación y que las patentes representan más bien a la investigación aplicada. La investigación básica no produce patentes, al menos de forma inmediata.

De todos modos, todas estas reflexiones no restan valor alguno a este indicador y más en una época como la actual, donde los recursos económicos son limitados y la universidad necesita disponer de fuentes de ingresos alternativos. También ha de justificar en mayor medida su papel en la sociedad, demostrando que contribuyen a su evolución y que de alguna manera rentabilizan el apoyo que ésta le proporciona. Azagra propone dos vías para reforzar las políticas de colaboración con las empresas: ampliar las fuentes de financiación de las universidades, y fomentar el rendimiento y la competitividad de las investigaciones. Para medir este segundo objetivo propone calcular la ratio existente entre el número de patentes elaboradas en las universidades y que generan negocio y el total de patentes que generan negocio (Azagra-Caro, 2009).

\section{Patentes de las universidades españolas en 2008}

Se pretende por un lado, mostrar cuáles son las universidades españolas con mayor producción de patentes, y por otro contrastar esa distribución con los resultados de alguno de los rankings anteriormente citados para reforzar nuestra idea. Este análisis no pretende ir más allá, ya que somos plenamente conscientes de que al reducir la temporalidad a un único año (2008) las conclusiones a extraer son limitadas, y más en un campo tan complicado en su gestión como es el del registro de avances o innovaciones en la investigación. También somos partícipes de que la gestión de una patente se lleva a cabo en un espacio de tiempo indeterminado, no pudiéndose establecer un tiempo medio porque cada caso es muy particular. De todos modos, este retraso también suele existir en la publicación de artículos en revistas científicas o en la publicación de tesis doctorales en repertorios o en otro tipo de publicaciones (manuales, monografías, etc.) y no afecta a la realización 
de rankings. Por tanto, pensamos que no ha de incidir considerablemente en el estudio.

\section{"La investigación básica se mide en los índices de impacto y la investigación aplicada en términos de patentes e inversión en I+D"}

Tomando como fuente la base de datos European patents fulltext ${ }^{2}$ se obtienen 240 patentes nacionales concedidas a universidades y hospitales universitarios españoles a lo largo del año 2008. Para su clasificación se toman los niveles básicos de la Clasificación internacional de patentes ${ }^{3}$ en su versión 2008.04 para establecer las distintas áreas de aplicación ${ }^{4}$ de las patentes y ver su distribución porcentual según las materias principales (tabla 1).

La categoría Química y metalurgia (C), seguida de Necesidades corrientes de la vida (A) y Física (G), presentan una mayor frecuencia en asignación de patentes y agrupan el $76 \%$ del conjunto global. Estos valores coinciden en gran medida con los aportados por González-Albo y Zulueta-García (2007) correspondientes a un período anterior de tiempo, por lo que esta distribución parece estar más o menos estabilizada. Como los niveles específicos de la clasificación asignados eran demasiado detallados y no permitían extraer resultados comparables, se han agrupado al segundo nivel de la clasificación antes citada ${ }^{5}$ (tabla 2).

Estas 11 materias principales representan el $73 \%$ del total. La distribución de patentes por universidades queda reflejada en la tabla 3.

Como era de esperar, la mayoría de las patentes se concentran en universidades públicas, organismos

\begin{tabular}{|l|c|r|}
\hline \multicolumn{1}{|c|}{ Categoría } & \multicolumn{1}{c|}{$\begin{array}{c}\text { No } \\
\text { ocurrencias }\end{array}$} & \% \\
\hline $\begin{array}{l}\text { A. Necesidades corrientes de } \\
\text { la vida }\end{array}$ & 94 & 24,4 \\
\hline $\begin{array}{l}\text { B. Técnicas industriales } \\
\text { diversas }\end{array}$ & 47 & 12,2 \\
\hline C. Química, metalurgia & 122 & 31,7 \\
\hline D. Textiles, papel & 1 & 0,3 \\
\hline E. Construcciones fijas & 11 & 2,9 \\
\hline $\begin{array}{l}\text { F. Mecánica, iluminación, } \\
\text { calefacción, etc. }\end{array}$ & 10 & 2,6 \\
\hline G. Física & 77 & 20,0 \\
\hline H. Electricidad & $\mathbf{2 3}$ & 6,0 \\
\hline TOTAL ${ }^{4}$ & $\mathbf{3 8 5}$ & $\mathbf{1 0 0 , 0}$ \\
\hline
\end{tabular}

Tabla 1. Distribución del número y porcentaje de las 240 patentes por materias principales de la CIP más dedicados a la investigación que las privadas, más orientadas hacia la docencia (aunque sí parece excesiva la gran diferencia existente). En muchos casos las universidades llevan a cabo el trabajo junto con otros organismos de investigación (el caso más frecuente es el CSIC con 43 participaciones) y con empresas. En este caso, el grado de coincidencia con la distribución de González-Albo-Manglano y Zulueta-García (2007) es algo menor (un 50\% entre las 12 primeras instituciones), algo normal porque estamos comparando un año con una distribución media de 20 años correspondientes a un período anterior de tiempo.

\section{"Los científicos inventores tienden a publicar mucho más que los no inventores"}

\section{Contraste de resultados.}

Si se comparan las universidades que tienen 10 o más patentes concedidas con aquellas que aparecen en los resultados entre las primeras 12 posiciones (doce son precisamente aquellas que tienen 10 o más patentes) de los parámetros estudiados por Buela-Casal et al. (2009), se obtienen los datos que se muestran en la tabla 4.

Al menos la mitad de las universidades a las que más patentes han concedido en el año 2008 (Politécni-

\begin{tabular}{|c|c|c|}
\hline $\begin{array}{l}\text { Códi- } \\
\text { go }\end{array}$ & Descripción & $\begin{array}{l}\text { Frecuen- } \\
\quad \text { cia }\end{array}$ \\
\hline A61 & $\begin{array}{l}\text { Ciencias médicas o veterinarias, } \\
\text { higiene }\end{array}$ & 67 \\
\hline G01 & Metrología, ensayos & 47 \\
\hline $\mathrm{C} 12$ & $\begin{array}{l}\text { Bioquímica, cerveza, bebidas } \\
\text { alcohólicas, vino, vinagre, micro- } \\
\text { biología, enzimología, técnicas de } \\
\text { mutación o de genética }\end{array}$ & 46 \\
\hline $\mathrm{CO}$ & Química orgánica & 36 \\
\hline B01 & $\begin{array}{l}\text { Procedimientos o aparatos físicos o } \\
\text { químicos en general }\end{array}$ & 23 \\
\hline A23 & $\begin{array}{l}\text { Alimentos o productos alimenti- } \\
\text { cios, su tratamiento, no cubierto } \\
\text { por otras clases }\end{array}$ & 12 \\
\hline H01 & Elementos eléctricos básicos & 12 \\
\hline A01 & $\begin{array}{l}\text { Agricultura, silvicultura, cría, caza, } \\
\text { captura, pesca }\end{array}$ & 11 \\
\hline G02 & Óptica & 10 \\
\hline $\mathrm{C} 01$ & Química inorgánica & 9 \\
\hline \multirow[t]{2}{*}{ G06 } & Cómputo, cálculo, conteo & 9 \\
\hline & TOTAL & 282 \\
\hline
\end{tabular}

Tabla 2. Distribución de patentes por materias secundarias CIP 
ca de Valencia, Barcelona, Autónoma de Madrid, Granada y Politècnica de Catalunya), se encuentran en las primeras posiciones en la mitad o más de los criterios empleados en el trabajo de referencia de Buela-Casal et al. (2009), pudiendo llegar a aventurar, con cierta lógica, que la patente agrupa a su alrededor gran parte del resto de los criterios utilizados. Este nivel de coincidencia, en torno al 50\%, parece indicar una correlación más fuerte si se ampliasen los períodos de tiempo del análisis. Para ello sería necesario que el trabajo de Buela-Casal se repitiera en el tiempo y realizar de nuevo el contraste. Muchas veces segmentar la actividad cien-

\begin{tabular}{|c|c|c|}
\hline Centro & $\begin{array}{c}\text { Pa- } \\
\text { tentes } \\
2008\end{array}$ & $\%$ \\
\hline Universitat Politécnica de Valencia & 27 & 10,88 \\
\hline Universidad del País Vasco & 20 & 8,06 \\
\hline Universidad Politécnica de Madrid & 19 & 7,66 \\
\hline Universidad de Zaragoza & 18 & 7,25 \\
\hline Universitat de Barcelona & 14 & 5,64 \\
\hline Universidad de Sevilla & 15 & 6,04 \\
\hline Universidad Complutense de Madrid & 15 & 6,04 \\
\hline $\begin{array}{l}\text { Universidad de Santiago de } \\
\text { Compostela }\end{array}$ & 13 & 5,24 \\
\hline Universidad Autónoma de Madrid & 12 & 4,83 \\
\hline Universidad de Granada & 12 & 4,83 \\
\hline Universitat Politècnica de Catalunya & 11 & 4,43 \\
\hline Universidad de Cádiz & 10 & 4,03 \\
\hline Universidad de Castilla-La Mancha & 6 & 2,41 \\
\hline Universidad de Málaga & 6 & 2,41 \\
\hline Universitat Autònoma de Barcelona & 6 & 2,41 \\
\hline Universitat d'Alacant & 5 & 2,01 \\
\hline Universidad de Córdoba & 4 & 1,61 \\
\hline Universidad de Murcia & 4 & 1,61 \\
\hline Universitat Jaume I & 4 & 1,61 \\
\hline Universidad Miguel Hernández & 4 & 1,61 \\
\hline Universidad de Salamanca & 3 & 1,20 \\
\hline Universidad de Valencia & 3 & 1,20 \\
\hline Universidad de La Laguna & 3 & 1,20 \\
\hline Universidad de Oviedo & 2 & 0,80 \\
\hline Universidad Pablo de Olavide & 2 & 0,80 \\
\hline Universitat de Les Illes Balears & 2 & 0,80 \\
\hline Universidad de Mondragón & 1 & 0,40 \\
\hline Universidad Antonio de Nebrija & 1 & 0,40 \\
\hline Universidad de Alcalá & 1 & 0,40 \\
\hline Universidad de Jaén & 1 & 0,40 \\
\hline Universidad Pública de Navarra & 1 & 0,40 \\
\hline Universitat de Girona & 1 & 0,40 \\
\hline Universitat Pompeu-Fabra & 1 & 0,40 \\
\hline Universitat Rovira i Virgili & 1 & 0,40 \\
\hline
\end{tabular}

Tabla 3. Distribución de patentes por universidad tífica por años introduce sesgos debido a los plazos de publicación de resultados o de registro de invenciones, que no tienen por qué coincidir. Analizar esta correlación a lo largo de distintos períodos de tiempo (quinquenios, décadas, períodos de años correspondientes a planes nacionales de I+D, etc.) contribuiría a homogeneizar los resultados y corregir situaciones algo contradictorias en nuestro contraste inicial: universidades que consiguen patentes año tras año no se encuentran entre las primeras que publican artículos ISI o reciben ayudas públicas de los planes I+D (como ha sido el caso de las universidades del País Vasco o Politécnica de Madrid en nuestro análisis).

\section{"Las categorías Química y metalurgia (C), seguida de Necesidades corrientes de la vida (A) y Física (G) agrupan el $76 \%$ de las patentes"}

El indicador más coincidente es el de becas FPU, algo lógico porque la obtención de una patente implica la realización de una importante serie de actividades previas de investigación en un grupo de trabajo. A medida que se va consolidando ese grupo de investigación y por la vía de los planes de I+D+i resulta más sencillo conseguir la incorporación de personal investigador a los proyectos en curso, lo que favorecerá posteriormente la realización de tesis doctorales y aumentará el número de artículos científicos publicados en revistas de impacto.

\section{"Varios años después de la presentación del invento se sigue observando actividad científica alrededor de la patente"}

\section{Reflexión final}

Si bien se detectan indicios de una posible correspondencia entre el número de patentes concedidas y otros indicadores de la actividad científica, se está aún lejos de confirmar esa tendencia, aunque lo lógico es que sea cierta. Esto no haría más que reforzar nuestro convencimiento de la necesidad de tomarlas en consideración como otro criterio más a la hora de realizar los rankings de la actividad científica de las universidades, para que aporten una perspectiva más global y menos influenciada (sesgada en algunos casos) por la trascen- 


\begin{tabular}{|c|c|c|c|c|c|c|}
\hline $\begin{array}{l}\text { Universidades con } \\
\text { más de } 10 \text { patentes }\end{array}$ & $\begin{array}{l}\text { Artículos } \\
\text { ISI }\end{array}$ & Tramos & $\begin{array}{c}\text { Proyectos } \\
\text { I+D }\end{array}$ & $\begin{array}{c}\text { Tesis } \\
\text { doctorales }\end{array}$ & Becas FPU & $\begin{array}{l}\text { Doctorados } \\
\text { mención calidad }\end{array}$ \\
\hline Politécnica de Valencia & $x$ & & & $x$ & $x$ & \\
\hline \multicolumn{7}{|l|}{ País Vasco } \\
\hline \multicolumn{7}{|l|}{ Politécnica de Madrid } \\
\hline \multicolumn{7}{|l|}{ Zaragoza } \\
\hline Barcelona & $x$ & $\mathrm{x}$ & & & $x$ & \\
\hline \multicolumn{7}{|l|}{ Sevilla } \\
\hline Complutense & & & & $x$ & $x$ & \\
\hline Santiago de Compostela & & & & & $x$ & \\
\hline Autónoma de Madrid & $x$ & $x$ & $x$ & $x$ & $x$ & \\
\hline Granada & & $x$ & & & $x$ & $x$ \\
\hline Politècnica de Catalunya & $x$ & & $x$ & $x$ & & $x$ \\
\hline Cádiz & & & & & & \\
\hline
\end{tabular}

Tabla 4. Contraste de resultados con el trabajo de Buela-Casal et al. (2009)

dencia de los índices de revistas con factor de impacto (aspecto éste ya muy debatido en la literatura especializada y que no es necesario recordar).

También somos conscientes de que este tipo de trabajos debe abordarse en series temporales mayores de un año y analizando de forma global todo el ciclo de vida de la actividad investigadora que da origen a la patente: petición del proyecto, concesión, composición del equipo investigador solicitante, puesta en marcha de la actividad científica, incorporación de nuevos recursos humanos y materiales que se aportan, elaboración de la memoria final, publicaciones y asistencia a congresos, elaboración de tesis doctorales, otras publicaciones derivadas y el posterior proceso de explotación de la patente por parte de la universidad (bien por acuerdo de colaboración con empresas o por medio de la constitución de una spin-off).

En cierta medida todo lo anterior ya se hace con los productos de investigación básica (el artículo científico preferentemente) y es posible que haya llegado la hora de hacerlo con los productos de la investigación aplicada, sin olvidar la influencia (positiva o negativa) que sobre las patentes han tenido y pueden tener las políticas puestas en práctica tanto en el ámbito español como comunitario. Todo ello nos permitiría disponer de un nuevo elemento a considerar en la planificación y en el reconocimiento de la actividad científica llevada a cabo en el seno de nuestras Universidades.

\section{Notas}

1. SCImago (2007). SJR, SCImago Journal \& Country Rank.

http://www.scimagojr.com

2. La base de datos European Patents Fulltext cubre todas las patentes europeas publicadas desde la apertura de la Oficina Europea de Patentes en 1978.

3. Esta clasificación puede consultarse en:

http://www.wipo.int/classifications/ipc/ipc8trans/es/ipcpub/

?lang $=e s \&$ menulang $=E S$
4. Hay patentes que tienen varias áreas de aplicación; se ha tomado nota de todas ellas, por lo que salen 385 áreas para 240 patentes.

5. Si se producía el caso de que una patente tuviera varias subcategorías asignadas sólo hemos contabilizado una por categoría, es decir si una patente tenía asignadas: C22C-0009/00 y C22C-0001/02, se consiga como ${ }^{\circ}$ de ocurrencias 1 para $\mathrm{C} 22$.

\section{Referencias}

50 carreras: curso 2009/2010. Madrid: El mundo, 2009. http://www.elmundo.es/especiales/2009/05/50carreras/concertados.html

Azagra-Caro, Joaquín M. La contribución de las universidades a la innovación. Tesis doctoral. Valencia: Universitat, 2004.

http://www.tesisenxarxa.net/TESIS_UV/AVAILABLE/TDX-0518105141320//azagra.pdf

Azagra-Caro, Joaquín M. Recognising the value of business patents with university inventors. Sevilla: JCR - Institute for Prospective Technological Studies, 2009.

http://cordis.europa.eu/erawatch/index.cfm?fuseaction=home. downloadFile \& fileID $=875$

Bordons, María; Zulueta, María-Ángeles. "Evaluación de la actividad científica a través de indicadores bibliométricos". Revista española de cardiología, 1999, v. 52, n 10. pp. 790-800.

http://www.doyma.es/cardio/ctl_servlet?_f=40\&ident $=190$

Buela-Casal, Gualberto; Bermúdez, María de la Paz; Sierra, Juan-Carlos; Quevedo-Blasco, Raúl; Castro, Ángel. "Ranking de 2008 en productividad en investigación de las universidades públicas españolas". Psicothe$m a, 2009$, v. 21, n. 2, pp. 304-312.

http://www.psicothema.com/pdf/3631.pdf

Buesa, Mikel; Heijs, Joost; Kahwash, Omar. La calidad de las universidades es España: elaboración de un índice multidimensional. Madrid: Comité Español de Automática, 2009.

http://www.cea-ifac.es/boletines/boletines/index31_archivos/ caliddunivrsidds0903CESparts123.pdf

Carayol, Nicolas. "Objectives, agreements and matching in science-industry collaborations: reassembling the pieces of the puzzle". Research policy, 2003, v, 32, n. 6, pp. 887-908.

European Commission. Delivering on the modernisation agenda for universities: education, research and innovation, $\mathrm{COM}(2006)$. Brussels: EC, 2006.

http://eur-lex.europa.eu/LexUriServ/LexUriServ.do?uri=COM:2006:0208: FIN:EN:PDF

González-Albo-Manglano, Borja; Zulueta-García, María-Ángeles. "Patentes domésticas de universidades españolas: análisis bibliométrico". Revista española de documentación científica, 2007, enero-marzo, v. 30, n. 1, pp. 61-90.

http://redc.revistas.csic.es/index.php/redc/article/view/369/373 
Hidalgo-Nuchera, Antonio. Los patrones de innovación en España a través del análisis de patentes: un análisis cualitativo en el período 1988 1998. Madrid: OEPM, 2003

Jiménez-Contreras, Evaristo; De-Moya-Anegón, Félix; Delgado-LópezCózar, Emilio. "The evolution of research activity in Spain: the impact of the National Commission for the Evaluation of Research Activity (Cneai)".

Research policy, 2003, v. 32, n. 1, pp. 123-142.

http://ec3.ugr.es/publicaciones/Research_policy.pdf

La universidad pública apuesta por la investigación; la privada, por la docencia. Madrid: El país, 25-05-2009.

http://www.elpais.com/articulo/sociedad/Universidad/publica/apuesta/ investigacion/privada/docencia/elpepusoc/20090525elpepisoc_2/Tes

Grupo Scimago. "La productividad ISI de las universidades españolas (2000-2004)". El profesional de la información, 2007, v. 16, n. 4, pp. 354358.

Sáiz-González, José-Patricio. Invención, patentes e innovación en la España contemporánea. Madrid: OEPM, 1999.

Schmookler, Jacob. Invention and economic growth. Boston: Harvard University Press, 1966

Spinak, Ernesto. "Indicadores cienciométricos". Ciência da informação, 1998 , v. 27 , n. 2 , pp. $141-148$ http://www.scielo.br/scielo.php?script=sci_arttext $\&$ pid $=S 0100-19651998$ $000200006 \& \ln g=e n \& n r m=i s o$

Van-Looy, Bart; Callaert, Julie; Debackere, Koenraad. "Publication and patent behaviour of academic researchers: conflicting, reinforcing or merely co-existing?" Research policy, 2006, v. 35, pp. 596-608

https://lirias.kuleuven.be/bitstream/123456789/101491/1/ publication+and+patent.pdf

Villanueva Kurczyn, Fernanda. "Divulgación científica: patentes vs. artículos”. Hypathia: Revista de divulgación científica, 2008, n. 27.

http://hypatia.morelos.gob.mx/index2.php?option=com_content\&do_ $p d f=1 \& i d=472$

Francisco-Javier Martínez-Méndez, Juan-Antonio Pastor-Sánchez y Rosana López-Carreño. Departamento de Información y Documentación, Universidad de Murcia.

javima@um.es

pastor@um.es

rosanalc@um.es

\section{Revista científica y profesional en español líder en el área de información, bibliotecas y nuevas tecnologías de la información.}

El profesional de la información

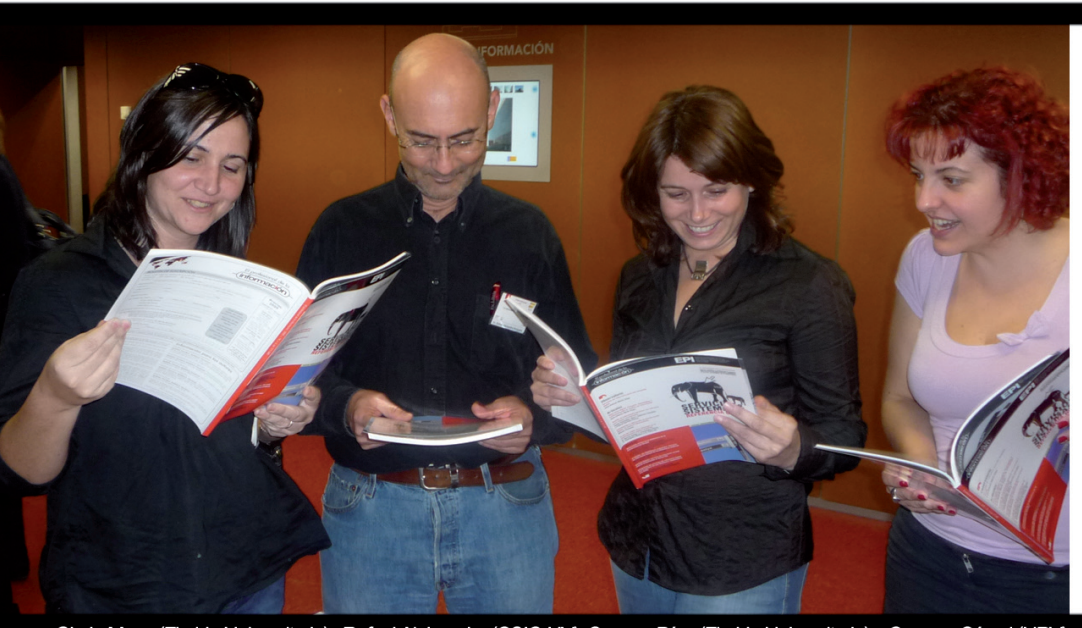

Gloria Moya (Florida Universitaria), Rafael Aleixandre (CSIC-UV), Susana Díaz (Florida Universitaria) y Carmen Cárcel (UPV

La 1a indexada porScience Citation Index

(Thomson ISI), y Scopus (Elsevier), además de otras 27 bases de datos y servicios bibliográficos.
Enero 2010 Sector editorial

Marzo 2010 Publicidad y comunicación empresarial

Mayo 2010 Información bio-médica

Julio 2010 Arquitectura de la información

Sept 2010 Cooperación de bibliotecas en red

Nov 2010 Medios de comunicación en intemet

Enero 2011 Psicología y sociología de la información

Marzo 2011 Archivos administrativos e intranets

Mayo 2011 Información de las administraciones públicas

\section{WwW.elprofesionaldelainformacion.com}

